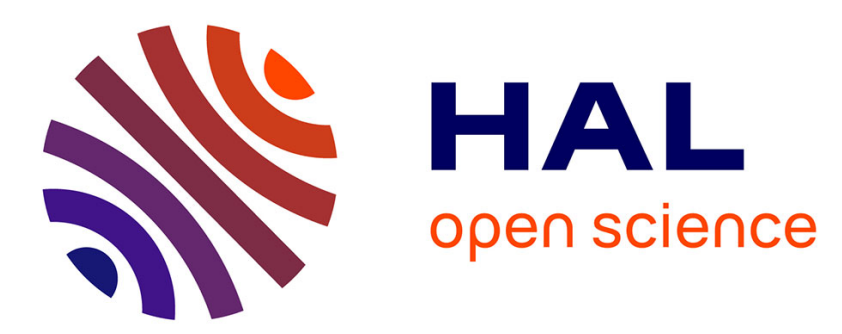

\title{
A DEM/FFT approach to simulate the effective thermal conductivity of granular media
}

Tristan Calvet, Jean-Mathieu Vanson, Renaud Masson

\section{To cite this version:}

Tristan Calvet, Jean-Mathieu Vanson, Renaud Masson. A DEM/FFT approach to simulate the effective thermal conductivity of granular media. International Journal of Thermal Sciences, 2022. hal-03427476

\section{HAL Id: hal-03427476 https://hal.science/hal-03427476}

Submitted on 14 Nov 2021

HAL is a multi-disciplinary open access archive for the deposit and dissemination of scientific research documents, whether they are published or not. The documents may come from teaching and research institutions in France or abroad, or from public or private research centers.
L'archive ouverte pluridisciplinaire HAL, est destinée au dépôt et à la diffusion de documents scientifiques de niveau recherche, publiés ou non, émanant des établissements d'enseignement et de recherche français ou étrangers, des laboratoires publics ou privés. 


\title{
A DEM/FFT approach to simulate the effective thermal conductivity of granular media
}

\author{
Tristan Calvet, Jean-Mathieu Vanson, Renaud Masson* \\ CEA, DES, IRESNE, DEC, Cadarache F-13108 Saint-Paul-Lez-Durance, France
}

\begin{abstract}
A numerical method to compute the Effective Thermal Conductivity (ETC) of granular media surrounded by a stagnant fluid is presented. Based on the geometry and size of grains, a Representative Volume Elements (RVE) of the granular media is created using the Discrete Element Method (DEM) while the ETC of this RVE is estimated with Fast Fourier Transform (FFT) computations.

To bridge the gap between the DEM and the FFT, a discretization algorithm (voxelisation of convex polyhedra) was developed. Since their physical properties are poorly defined, "fuzzy" voxels associated with solid-gas interfaces or solid-solid contacts, domains inherent in a granular medium, are clearly identified during this step. The assignment of extreme properties to these voxels, assuming negligible radiation, allows to establish lower and upper ETC bounds.

This methodology is applied to granular media of Uranium dioxide particles immersed in stagnant Helium; packed beds for which experimental data are available in the literature. First, the effect of RVE and discretization sizes on the simulated ETC are investigated and optimal values of RVE size and discretization ratio are defined. Next, the significant deviation of simulated DEM-FFT ETC bounds, observed at high solid to gas conductivity contrasts, exhibits the key role of interfaces on the computed ETCs. An improved modelling of solid-gas interfaces based on the Knudsen effect and a modelling correction for solid-solid interfaces is therefore proposed. The new modelling is shown to adequately bound and estimate the ETC of considered granular media at high $(\sim 100)$ solid to gas thermal conductivity contrasts.
\end{abstract}

Keywords: Effective Thermal Conductivity, Granular media, Representative Volume Element, Discrete Element Method, Fast Fourier Transform, Knudsen effect

\section{Introduction}

Modelling the equivalent macroscopic properties of heterogeneous media is an important issue for many applications, especially for the ones involving granular media. The heterogeneous, complex microstructure of granular media often implies chemical, electrical or thermal effective behaviours commonly driven by, but not solely, respective phase properties, interfaces between phases and property ratio of phases. While considering the thermal properties of packed beds constituted of particles surrounded by a gaseous phase, the thermal fluxes in each phase or passing between phases compete altogether making contacts or near-contacts of

\footnotetext{
${ }^{*}$ Corresponding author

Email addresses: t. calvet19@imperial .ac.uk (Tristan

Calvet), jean-mathieu.vanson@cea.fr (Jean-Mathieu Vanson), renaud.masson@cea.fr (Renaud Masson)
}

particles key in the prediction of thermal effective properties, particularly at high contrast of each phase properties. The prediction of the effective thermal conductivity is important in order to model materials for which conducting experimental measurements is either impossible or too complex and expensive (e.g. nuclear materials).

For thermal conduction problems and considering granular media with interstitial stagnant fluid, the Wiener [1], Maxwell-Eucken [2] theoretical works provide bounds of the effective thermal conductivity (ETC) of the considered heterogeneous material. However, these bounds deviate significantly which prevents their application to the estimation of effective thermal properties of granular media. Alternatively, theoretical models [3] (packed beds with locally spherical contacts) or models based on an uni-dimensional representation of the heat flux have also been proposed, see for example $[4,5,6]$. However, such models do no

November 14, 2021 


\begin{tabular}{|c|c|c|c|}
\hline \multicolumn{4}{|c|}{ Nomenclature } \\
\hline$K_{n}$ & Knudsen number (-) & $p$ & Porosity of the solid particles (-) \\
\hline$T$ & Temperature $(K)$ & $r_{e q}$ & Mean size of the particles $(m)$ \\
\hline$\beta$ & Thermal polarizability (-) & \multicolumn{2}{|c|}{ Abbreviations } \\
\hline$\lambda$ & Thermal conductivity $\left(\mathrm{W} \mathrm{m}^{-1} \mathrm{~K}^{-1}\right)$ & $D E M$ & Discrete Element Method \\
\hline$\sigma$ & Stefan-Boltzmann constant $\left(\mathrm{kg} \mathrm{K}^{-4} \mathrm{~s}^{-3}\right)$ & ETC & Effective Thermal Conductivity \\
\hline$j$ & Heat flux $\left(W m^{-2}\right)$ & $F F T$ & Fast Fourier Transform \\
\hline$\varepsilon$ & Emissivity (-) & $R V E$ & Representative Volume Element \\
\hline$\varphi$ & Porosity of the granular media $(-)$ & \multicolumn{2}{|c|}{ Subscripts } \\
\hline$d_{m}$ & Kinetic molecular diameter $(m)$ & $e$ & Effective \\
\hline$d l$ & Size of the voxels $(m)$ & $g$ & Gas \\
\hline$k_{b}$ & Boltzmann constant $\left(\mathrm{kg} \mathrm{s}^{-1} \mathrm{~K}^{-1}\right)$ & $s$ & Solid \\
\hline
\end{tabular}

take into account the key microstructural parameters related to the considered granular material. As a result, these models are generally fitted on experimental data. Considering the interstitial stagnant fluid as a perfect thermal insulator, the so-called Discrete Element Method (DEM) has been used to simulate Representative Volume Elements (RVE) of granular media as well as the effective conductivity of these RVEs (see for example [7] and [8]). To account for the conductivity of the stagnant fluid surrounding solid particles with the DEM, Yun [9] proposed to model heat transfers at interfaces using resistor models when the particles have a spherical shape. Furthermore, numerical methods combining the Discrete Elements and the Finite Element Methods have also been proposed (see for example [10]). These methods aim at modelling the effect of key microstructural parameters such as the size, shape and spatial distribution of the solid particles on the effective thermal conductivity. Nevertheless, the modelling of the heat flux at the interfaces between the solid and gaseous phases remains often arbitrary. Recently, and using a DEM approach, Moscardini [11] proposed to take into account the influence of the interstitial gas pressure and temperature on the thermal contact conductance between particles. However, this modelling of solid-fluid interfaces is not adequate for complex, non-spherical fragment geometries and requires improvements. Overall, interfaces in packed beds have a prominent importance in the simulation of the ETC of packed beds and their modelling remains a challenge which requires further research and enhance- ments.

The use of Fast Fourier Transform (FFT) instead of Finite Elements offers some advantages. It relies on a regular grid which is easier and faster to compute than a non regular finite element mesh. Moreover, the computations are efficiently accelerated thanks to parallelisation that is, in this case, easier to implement. In [12], the microstructure model was directly obtained by tomography. Here, we propose to combine the DEM to generate a RVE of the granular medium. Indeed, the DEM is a powerful technique to generate packed beds at a given density. Then, we propose to use FFT to simulate the effective property of the RVE. A discretization algorithm was used to bridge the gap between the DEM and the FFT computations.

This paper is organized as follows. In section 2, reference packed beds experimental data (Uranium dioxide granular packing immersed in Helium) $[13,14]$ obtained from the literature are presented. Materials properties from the solid and gas phases are defined clearly and the potential effects of radiation and the Knudsen effect on the ETC of considered media are discussed. In section 3, the new methodology associating the DEM and FFT methods is presented. The two bounds and the estimate delivered by this new methodology are applied to the prediction of the effective conductivity of an idealized dispersion of spheres at the end of section 3. In section 4 , the real packed beds presented in section 2 are replicated used the DEM while DEM-FFT simulations of the 
ETC of these experimental beds are compared to experimental results. The role of the solid-solid and solid gas interfaces as well as the Knudsen effect on the simulated ETC are deeply studied. New DEM-FFT estimates are then derived. In section 5 , the best bounds and estimates of the ETC predicted by the DEM-FFT approach are finally compared to experimental results.

\section{Materials}

\subsection{Experimental data}

Experimental data are extracted from [14] (NACA experiments) and have been obtained with a radial apparatus during a steady regime. The gas volume fraction reaches nearly $40.5 \%$. The size distribution is: $60 \%$ of particles with diameters between 20 and $80 \mu \mathrm{m}$ and $40 \%$ between 80 and $120 \mu \mathrm{m}$. The solid particles and the gaseous phase are Uranium dioxide and Helium, respectively. In [14], no information about the shape of the powder particles is given. The thermal properties of Uranium dioxide and Helium depend mainly on temperature and are described below (see section 2.2).

We extracted data from the fitted curves computed by Boegli et al.. These data are reported on Table 1 . The relative deviation of the experimental data is around $5-10 \%$. At $472 K$, we have also reported on Table 1 the experimental data provided by Hall [13]. The packed beds studied by Hall [13], although characterised by various uniform size distributions of $\mathrm{UO}_{2}$ spheres (ranging from 80 to $800 \mu \mathrm{m}$ in size) with densities of $60.5 \%$, provide a range of relevant experimental values. We take into account in Table 1 the experimental value by Hall for a packed bed of $80 \mu \mathrm{m}$ spheres, this packed bed being most representative of our owns. The remaining experimental values obtained for bigger spheres were considered to define a relative experimental error margin of 5\%. As explained in the next section 2.2, all these considered ETC measurements were taken under high helium pressure (Boegli: 0.41 - $0.94 \mathrm{MPa}$, Hall: $\sim 1.7$ $\mathrm{MPa}$ ) in order to overcome the thermal interface resistance existing between the gas and the solid, discussed in the subsection 2.4 .

\subsection{Material properties}

The thermal conductivity of Uranium dioxide is expressed as a function of temperature $(T)$ and porosity ( $p)$ according to [15]:

$$
\lambda_{s}=\frac{1-p}{1+0.5 p}\left[\frac{1}{A+B T}+C T^{-2} e^{\frac{D}{T}}\right],
$$

where $A=0.0375 \mathrm{~K} . \mathrm{m} . \mathrm{W}^{-1}, B=2.165 \times 10^{-4} \mathrm{~m} . \mathrm{W}^{-1}$, $C=4.715 \times 10^{9} \mathrm{~W} \cdot \mathrm{m}^{-1} . K, D=-16361 K$. For the next experimental data, the porosity equals $2 \%(p=0.02)$. The thermal conductivity of Helium is expressed as a polynomial function of the temperature $(T)[16]$ :

$$
\lambda_{g}=E+F T-G T^{2}+H T^{3},
$$

where $E=4.76 \times 10^{-2} W \cdot m^{-1} \cdot K^{-1}, F=3.62 \times$ $10^{-4} W \cdot m^{-1} \cdot K^{-2}, G=6.18 \times 10^{-8} W \cdot m^{-1} \cdot K^{-3}, H=$ $7.18 \times 10^{-12} W \cdot m^{-1} \cdot K^{-4}$.

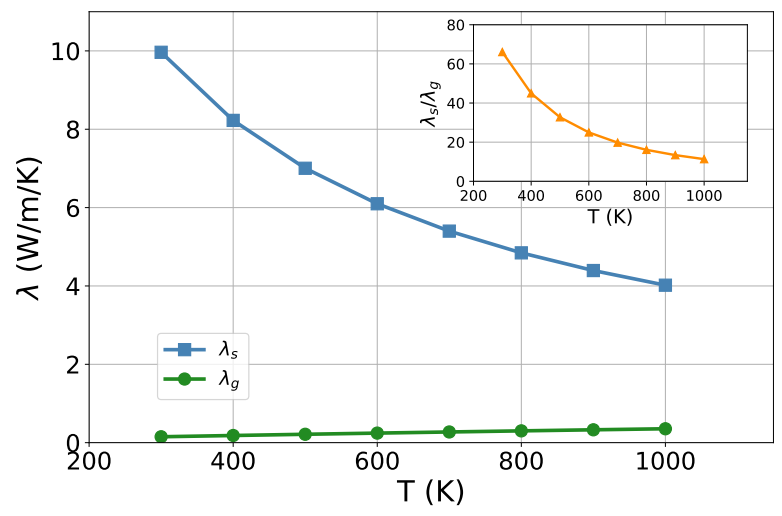

Figure 1: Effect of temperature on the thermal conductivity of helium $\left(\lambda_{g}\right)$, uranium dioxyde $\left(\lambda_{s}\right.$ with inernal porosity $\mathrm{p}=0.02$ and Burnup $=0 . \mathrm{GW} / \mathrm{D} / \mathrm{tM})$ and solid to gas conductivity contrast

The evolution of the thermal conductivity of Helium $\left(\lambda_{g}\right)$ and $U_{2}\left(\lambda_{s}\right)$ as function of temperature, described by equations (1) and (2) are depicted in Fig. 1 as well as the resulting conductivity contrast $\left(\lambda_{s} / \lambda_{g}\right)$. As seen, temperature has opposite effect on the conductivities of Helium and $\mathrm{UO}_{2}$. Overall, the contrast is maximal at low temperatures and decreases progressively with temperature. The maximum observed contrast for relevant temperatures is less than 100 .

\subsection{Radiation contribution}

Radiation in packed beds may be a considerable mechanism for heat transfer notably at high temperatures. Inside our granular media, radiation occurs in the interstitial spaces between particles (called pores hereafter). The effect of radiation on the ETC depends upon the bed temperature, the size and shape of pores and upon radiation properties of the solid and gas phases (transparency, emissivity, absorption). In the fluid, radiation is in direct competition with the conduction heat mechanism characterised by the fluid thermal conductivity. A comparison between these mechanisms thus is needed to determine their relative importance. 


\begin{tabular}{|c|c|c|c|c|c|c|c|c|c|}
\hline Temperature $(K)$ & 366.5 & 422 & 472 & 477.5 & 533 & 588.5 & 644 & 755 & 866 \\
\hline Conductivity $(W / m / K)$ & 1.36 & 1.41 & 1.34 & 1.43 & 1.45 & 1.46 & 1.47 & 1.47 & 1.47 \\
\hline
\end{tabular}

Table 1: Experimental data extracted from [14] (NACA experiments) and from [13] (Hall experiments).

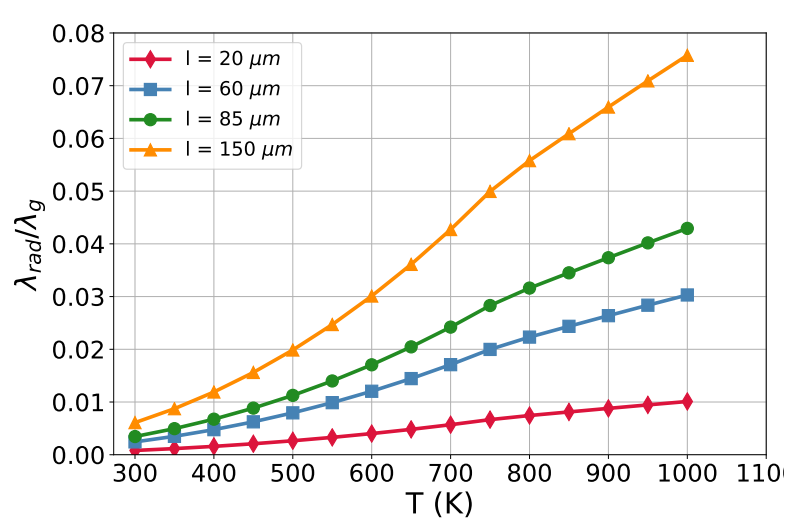

Figure 2: Effect of temperature and pore size (modelled as infinite parallel plates separated by a distance $l$ ) on the relative contribution of radiation to heat conduction in $\mathrm{He}$.

We consider that the conductivity due to radiation in packed beds is always lower than the radiant conductivity between infinite parallel plates separated by a distance $l$ equal to the size of pores. The pore size distribution for the considered granular beds is more deeply studied in section 4 with the maximum inscribed ball technique depicted in section 4.3. It is demonstrated that the diameter of pores is about $60 \mu \mathrm{m}$ for the considered granular media (see Fig. 14).

Assuming that the temperature difference between planes is small when compared to the average temperature, the thermal conductivity due to radiation between parallel infinite planes is the following:

$$
\lambda_{\text {rad }}=\frac{4 \epsilon \sigma l T^{3}}{2-\epsilon}
$$

where $T$ is the average temperature, $\sigma$ the StefanBoltzmann constant $\left(\mathrm{kg} \mathrm{K}^{-4} \mathrm{~s}^{-3}\right)$ and $\epsilon$ the emissivity (-) of $\mathrm{UO}_{2}$.

Helium is considered to be a transparent gas and $\mathrm{UO}_{2}$ an opaque solid which has a decreasing emissivity with temperature [17]. Fig. 2 shows that even for the largest interstitial space between particles $(\approx 60 \mu \mathrm{m})$, radiant thermal conductivity will be less than three percent of the gas conductivity at the highest temperature $(1000 \mathrm{~K})$ considered in this study. The assumption of negligible radiant heat transfer is therefore relevant for the packed beds considered here. Further discussion on the limited effect of radiation on the ETC through the analysis of thermal heat fluxes paths inside granular media RVEs is presented with the analysis of thermal heat maps (cf. section 4.2).

\subsection{Knudsen effect}

The influence of pressure on the effective thermal conductivity of helium was considered in accordance to the Knudsen effect [18]: as the mean free path of the gas molecules increases when the pressure decreases, this mean free path may reach the dimension of the gas layer between particles inducing an additional thermal resistance between particles. To quantify this potential Knudsen effect (also called "Smoluchowski effect" in [11]), the calculation of the ratio between this mean free path and the gas layer (Knudsen number) is needed, this number $K_{n}$ being given by:

$$
K_{n}=\frac{k_{b} T}{\sqrt{2} \pi d_{m}^{2} P L}
$$

where $k_{b}$ is the Boltzmann constant, $d_{m}$ the kinetic molecular diameter $\left(d_{m}=2.1510^{-10} \mathrm{~m}\right.$ for Helium according to [11]), $P$ the pressure and $L$ the dimension of confining spaces between particles.

The measurements of the effective conductivity reported by [14] were made under high helium pressure $(P \sim 1.7 \mathrm{MPa})$ overcoming this Knudsen effect. Experimental data reported in section 4.3 have been conducted in the temperature range $[300,1000 \mathrm{~K}]$.

In [14], the dimension of confining spaces between particles was chosen equal to $45 \mu \mathrm{m}$ (mean size of the particles). If this dimension equals the radius of the smallest particles $(10 \mu \mathrm{m})$, the Knudsen number varies in the considered range of temperature from 0.001 to 0.004 placing our packed beds in the lower part of the transition regime $\left(0.001 \leq K_{n} \leq 10.0\right)$ between the continuum regime (the heat transfer between particles is dominated by the gas conduction - see (2)) and the free molecule regime (the heat transfer between particles is dominated by the interactions of the gas molecule with the solid surfaces). Therefore, the Knudsen effect can not be fully neglected and will be studied in-depth in section 4.3. 


\section{Methods}

\subsection{Discrete Element Method}

The discrete element method is widely used to simulate the granular behavior of matter in many fields. It makes it possible to efficiently simulate the granular rearrangements taking into account the shape, the size of the particles as well as their mutual interactions. Here, the particles are considered rigid while their motion is calculated from the detection of their mutual contacts and the computation of the contact forces. The network of forces is solved by the implicit method of contact dynamics [19] using the software LMGC90 [20]. At each time step, the method is composed of three steps. The first step consists in detecting the contacts between particles. The second step aims at calculating the forces of the previously detected contacts and the third step consists in updating the position of the particles from the forces balance. Interactions between particles are governed by the Signorini conditions and by a Coulomb dry friction law (Fig. 3).

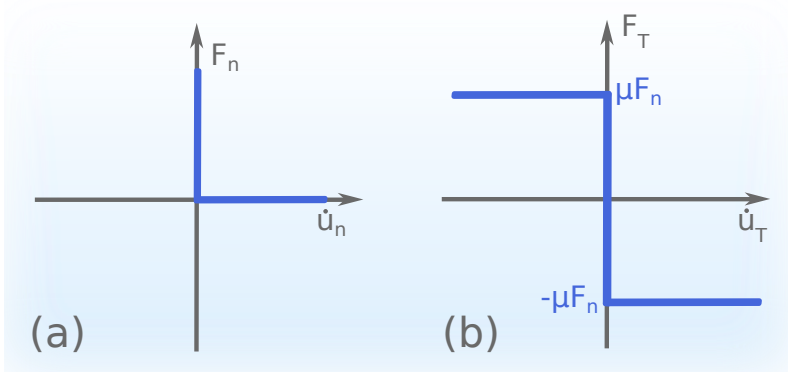

Figure 3: Contact law: Signorini Conditions (a) and Coulomb law (b) connecting respectively the normal force to the normal speed and the tangential force to the tangential velocity. $\mu$ is the friction coefficient.

We create each packing from a size distribution (defined in section 4) and a regular polyhedra shape having 20 faces. We first place the polyhedra in a box using a potential energy minimization algorithm (gravity). Then, we perform DEM computation of polyhedra motion under gravity with periodic boundary conditions over the $\mathrm{X}$ and $\mathrm{Y}$ axis and a fixed plane at $\mathrm{Z}=0$. We use a static Coulomb friction law (friction coefficient $=$ 0.2 ). The porosity has a first order influence on the ETC. We chose the Coulomb friction coefficient to control the porosity of the simulated packed beds and obtain porosity values close from NACA experiments[14]. When the kinetic energy tends to zero we cut the domain with two planes orthogonal to the $\mathrm{Z}$ axis to avoid side effects and keep it full over the periodic directions (X and $\mathrm{Y}$ axes). An example of DEM generated granular media is depicted in figure 4.

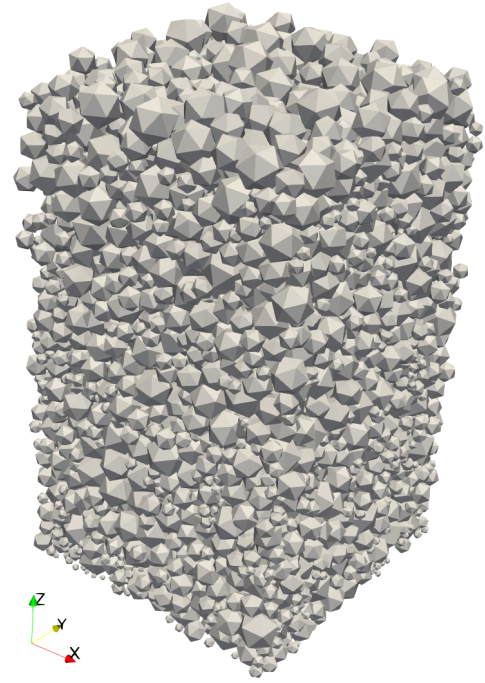

Figure 4: Granular media constituted of regular polyhedra (icosahedra) generated with the Discrete Element Method with $\mathrm{X}$ and $\mathrm{Y}$ axes periodic boundary conditions.

These DEM simulations yield a Representative Volume Elements (RVE) of the granular medium under consideration. A given RVE denoted by $V$ is made of two different homogeneous constituents (the solid particles and the fluid) with $\varphi$ the volume fraction of the fluid. $\left(\lambda_{s}, \lambda_{g}\right)$ denote the thermal conductivity of the two constituents.

The strain of the particles being not taken into account (rigid particles), the contact area between particles does not depend on their mechanical interactions. This approximation is consistent with the fact that the RVE considered hereafter will be stress-free at their boundary so that the residual force network (and the resulting contact area) remains negligible.

\subsection{Discretization of DEM RVEs}

The cartesian discretization needed to compute the ETC with the FFT method consists in a regular 3D grid, each subvolume of this grid being called a voxel (see section 3.3). To build this grid, an additional algorithm is needed to discretize the generated DEM packed beds into numerous voxels of size 'dl', differentiated by their respective phase index. This algorithm is depicted below.

As illustrated on Fig. 5, most voxels are fully defined by a solid or gas phase index and are thus assigned with 
solid or gas thermal conductivity. The remaining voxels, called hereafter fuzzy voxels, are solid-gas interface voxels as well as solid-solid contact voxels located at the contact interface between distinct polyhedra. All fuzzy s/g and fuzzy s/s voxels posses unknown equivalent thermal conductivity since they are constituted of either two distinct phases, and/or embedded with an illdefined thermal resistance resulting from the imperfect contact between fragments.

Solid-solid contact voxels (fuzzy s/s) either result from occasional surface to surface contacts and point contacts between particles usually coupled with minor bias from the DEM and/or from the discretization (seen notably in the construction of the upper bound described below). Assigning the solid thermal conductivity to solid-solid contact voxel creates unrealistic perfect thermal bridges between particles which may significantly increase the ETC of the RVE especially when the solid to gas conductivity ratio is high. To limit the impact of solid-solid contacts on the ETC, the discretization may be refined. However, taking into account a particle surface roughness, the discretization size (dl) should not be any smaller than twice the surface roughness of particles. Another proposed correction (cf. section 4.2 ), is the insulation of particles by a thin layer of gas equalling, in our case, to twice the surface roughness of particles (Mean estimate no-contact in Table 2). Although, this correction may lead to an underestimation of the media ETC, its implementation results in a improved modelling of solid-solid contacts.

Since the thermal conductivity of the solid phase is higher than the fluid phase conductivity in our case, assigning the conductivity of the solid to the fuzzy s/g voxels yields an upper bound for the effective thermal conductivity. Oppositely, assigning to the fuzzy s/g voxels the conductivity of the fluid yields a lower bound. This elementary result is the direct extension for conduction problems of the Strengthening theorem formulated by [21] for linear elastic heterogeneous materials.

Alternatively, the usual method consists in defining the conductivity of the fuzzy $\mathrm{s} / \mathrm{g}$ voxels regarding the position of the center of the cell of the cartesian mesh. This method, called "mean estimate" hereafter, yields estimates of the effective conductivity lying between the two bounds defined above.

\subsection{Fast Fourier Transform computations of the ETC and discretization of RVES}

In the steady-state regime with no source terms, the thermal field as well as the heat flux field (denoted by $\boldsymbol{j}$ )

\begin{tabular}{|c|c|c|}
\hline & $\lambda_{\text {fuzzy } s / g}$ & $\lambda_{\text {fuzzy } s / s}$ \\
\hline Upper Bound & $\lambda_{s}$ & $\lambda_{s}$ \\
\hline Lower Bound & $\lambda_{g}$ & $\lambda_{g}$ \\
\hline Mean estimate & $\lambda_{\text {center }}$ & $\lambda_{\text {center }}$ \\
\hline Mean estimate no-contact & $\lambda_{\text {center }}$ & $\lambda_{g}$ \\
\hline
\end{tabular}

Table 2: Values of the conductivities of fuzzy voxels defining the upper bound, the lower bound and the mean estimate.

Data: DEM outputs : position of all polyhedra and their respective nodes

Initialization : creation of global container (3D grid: $N_{x}, N_{y}, N_{z}$ ) considering discretization parameter ( $\mathrm{dl}$ ) and extreme positions of all polyhedron nodes;

\section{for each polyhedron $(\mathrm{p})$ do}

for each face (f) of polyhedron 'p' do Detect "fuzzy s/g" voxels situated on face 'f';

for each "fuzzy s/g" voxel (v) of face ' $f$ ' do

Assign value of volume fraction of solid phase to voxel 'v';

end

Detect all bulk voxels enclosed by "fuzzy s/g" voxels. Assign to bulk voxels the solid thermal conductivity;

end

end

if lower_bound_structure then
Assign to all "fuzzy s/g" voxels gas thermal conductivity

else

Assign to all "fuzzy s/g" voxels solid thermal conductivity

end

Selection of periodic RVE for effective thermal conductivity computation;

Algorithm 1: Voxelisation algorithm for DEM-FFT ETC bounds 


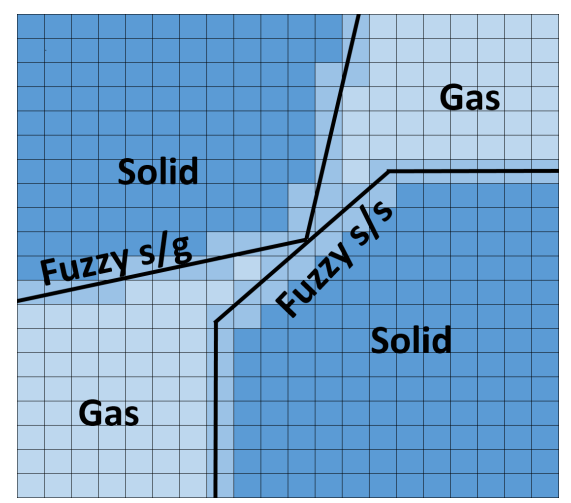

Figure 5: Voxelisation of a 2D structure composed of two polygons (outlined in black). Dark and light blue pixels respectively designate solid and fluid phase. Pixels in intermediate blue ("fuzzy" pixels) are ill-defined and possess unknown effective properties. Assigning to the "fuzzy" pixels extreme conductivity (either solid or gas conductivity) leads to RVE with lower or upper ETC bound. "Fuzzy" pixels are further subdivided into solid-gas interface "fuzzy s/g" pixels and solid-solid contact "fuzzy s/s" pixels.

are solutions of $(\boldsymbol{x} \in V)$ :

$$
\nabla \cdot \boldsymbol{j}(\boldsymbol{x})=\mathbf{0} \quad \text { with } \quad \boldsymbol{j}(\boldsymbol{x})=\lambda(\boldsymbol{x}) \boldsymbol{e}(T(\boldsymbol{x}))
$$

where the intensity vector field $\boldsymbol{e}$ is defined as the opposite of the thermal gradient $\boldsymbol{e}(T(\boldsymbol{x}))=-\nabla \cdot T(\boldsymbol{x})$. To solve the boundary value problem (5), we use the Fast Fourier Transform (FFT) method originally proposed by [22]. In this method, periodic boundary conditions are considered, the macroscopic intensity vector $\overline{\boldsymbol{e}}$ being prescribed in volume average (see [12]). Therefore, the unknown temperature and intensity fields have the general form:

$$
T(\boldsymbol{x})=T^{*}(\boldsymbol{x})-\overline{\boldsymbol{e}} \cdot \boldsymbol{x} \quad \text { and } \quad \boldsymbol{e}(T(\boldsymbol{x}))=\boldsymbol{e}\left(T^{*}(\boldsymbol{x})\right)+\overline{\boldsymbol{e}} .
$$

where $T^{*}(\boldsymbol{x})$ denotes a periodic field. If $\lambda_{0}$ denotes an arbitrary conductivity, the relation between the heat flux and the intensity vector reads:

$$
\boldsymbol{j}(\boldsymbol{x})=\lambda_{0}(\boldsymbol{x}) \boldsymbol{e}(T(\boldsymbol{x}))+\boldsymbol{\tau}(\boldsymbol{x})
$$

with the polarization field $\boldsymbol{\tau}(\boldsymbol{x})$ defined as:

$$
\boldsymbol{\tau}(\boldsymbol{x})=\left(\lambda(\boldsymbol{x})-\lambda_{0}\right) \boldsymbol{e}(T(\boldsymbol{x})) .
$$

The unknown intensity field satisfies the following integral equation:

$$
e\left(T^{*}(\boldsymbol{x})\right)+\Gamma_{0} \star \tau(\boldsymbol{x})=\mathbf{0}
$$

where $\star$ denotes the convolution product and $\boldsymbol{\Gamma}_{0}$ is the periodic Green-operator associated with $\lambda_{0}$. This operator being explicitly known in Fourier space, this last equation is solved in Fourier space:

$$
\boldsymbol{\xi} \neq 0: \hat{\boldsymbol{e}}(\boldsymbol{\xi})=-\hat{\Gamma}_{0}(\boldsymbol{\xi}) \cdot \hat{\boldsymbol{\tau}}(\boldsymbol{\xi}) \text { and } \hat{\boldsymbol{e}}(\mathbf{0})=\mathbf{0} .
$$

The iterative algorithm proposed by [23] and adopted here, uses alternately (6) and (7) in real and Fourier spaces. To compute the unknown temperature and intensity fields, the RVE $V$ is discretized into $n_{v o x}=$ $N_{x} \times N_{y} \times N_{z}$ regular cubic regions (the voxels introduced in the previous section 3.2), the unknown intensity field being chosen constant per voxel. As a result, the polarization is also constant per voxel and is computed in the real space. Thus, the polarization for any voxel $v$ is given by:

$$
\boldsymbol{\tau}_{v}=\left(\lambda_{v}-\lambda_{0}\right) \boldsymbol{e}_{v},
$$

where $\lambda_{v}$ is the conductivity assigned to the voxel $v$. As recalled in the previous section, the position of the center of the voxel defines generally the value of its conductivity which remains arbitrary when the considered voxel intercepts a solid-gas interface.

For an isotropic behavior, the effective thermal conductivity is a scalar denoted by $\lambda_{e}$ and can be computed by prescribing the macroscopic intensity in an arbitrary direction, for instance the $\boldsymbol{e}_{y}$ direction:

$$
\overline{\boldsymbol{e}}=\bar{e}_{0} \boldsymbol{e}_{y} \quad \Rightarrow \quad \lambda_{e}=\frac{<\boldsymbol{j} \cdot \boldsymbol{e}_{\boldsymbol{y}}>}{\bar{e}_{0}}
$$

where $<$. $>$ denotes the volume average on the RVE $V$, namely:

$$
<\boldsymbol{j}>=\frac{1}{n_{v o x}} \sum_{v=1}^{n_{v o x}} \lambda_{v} \boldsymbol{e}_{v} .
$$

Compared to the classical Finite Element method, the FFT method is able to handle a large number of degrees of freedom and can be accelerated by parallelization techniques (through this work, an OpenMP parallelization technique is used). In addition, the FFT method has the great advantage to have a Cartesian mesh being easier and faster to perform than a non regular classical finite element mesh.

\subsection{Validation of DEM-FFT bounds on a reference Body-Centered Cubic structure}

In order to test the reliability of the mean estimates and bounds defined above, we consider an ideal twophase microstructure where the solid phase is spheres arranged in a Body-Centered Cubic (BCC) configuration. For this periodic structure and a superconducting solid $\left(\lambda_{s} / \lambda_{g} \rightarrow \infty\right)$, the effective conductivity has already been computed exactly by [24] as a function of the solid volume fraction. For this analysis, a volume 
fraction of $53.6 \%$ is considered, while the effect of the discretization ratio for different solid to fluid conductivity ratio is analysed.

The BCC structure unit cell needed for FFT computations reduces to a cubic cell with one sphere at the center and one-eighth of spheres at the eight edges of this cube (the BCC crystalline mesh). Therefore, if $r$ denotes the radius of the spheres, the discretization ratio $(r / d l)$ can be chosen small enough to reach a volume fraction of fuzzy voxels close to zero. Indeed, for the highest contrast considered in this work $\left(\lambda_{s} / \lambda_{g}=100\right.$, see Fig. 1), the predicted effective conductivity as a function of this discretization ratio is reported on Fig 6. The evolution of the volume fraction of the fuzzy voxels with the discretization ratio is also reported on Fig 6. As the volume fraction of the fuzzy voxels decreases with the discretization ratio, the bounds and the estimate all converge to the theoretical effective conductivity of this BCC periodic micro-structure. For $r / d l>90$, the deviation between the mean estimate and the theoretical result is less than $1 \%$.

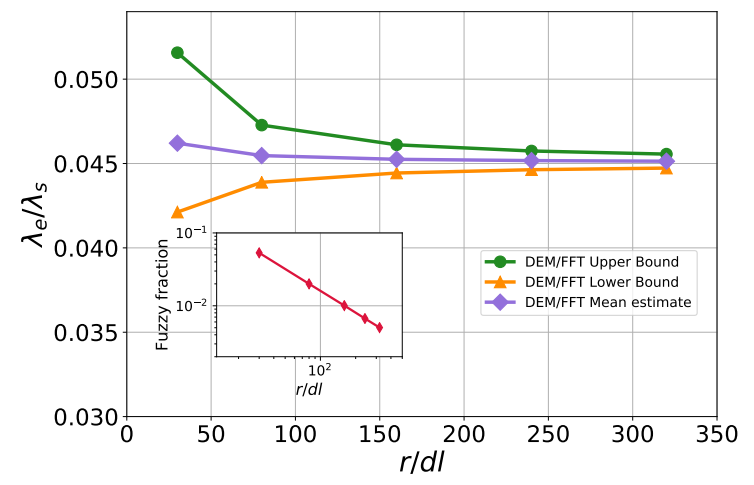

Figure 6: FFT computations of the effective conductivity of a solid made of identical spheres arranged on a periodic BCC array. Evolutions of the bounds and the estimate as a function of the discretization ratio $\left(\lambda_{s} / \lambda_{g}=100\right)$.

Additionally, the effect of the contrast on the ETC of the BCC structure is studied for a wide range of conductivity contrasts $\left(\lambda_{s} / \lambda_{g}\right)$. Consistently with the previous results, the theoretical results are derived with the "mean estimate" obtained by FFT and a discretization ratio $r / d l=320$. We will see later that to perform FFT computations on realistic granular media, a lower discretization ratio has to be adopted, namely $r_{e q} / d l$ ranging from 10 to 60 (average around 30). $r_{e q}$ is the equivalent radius of particles for the granular media considered. Thus to estimate the effect of this discretization ratio for the next predictions, we compare on Fig. 7 the FFT predictions obtained with $r / d l=30$ (bounds and estimate) to these reference results. The FFT lower bound and the FFT mean estimate give a good agreement with the reference solution. The FFT upper bound overestimates the effective conductivity since the increased amount of solid creates solid thermal bridges between the spheres (the spheres overlap). Even if the volume fraction of the fuzzy voxels remains quite low for this ideal structure $(\approx 5.3 \%$ for the considered value of the discretization ratio, namely $r / d l=30)$, the gap between the FFT lower and upper bounds is not negligible $(\approx 22 \%)$ at a high conductivity contrast $\left(\lambda_{s} / \lambda_{g}\right)$ of 100 .

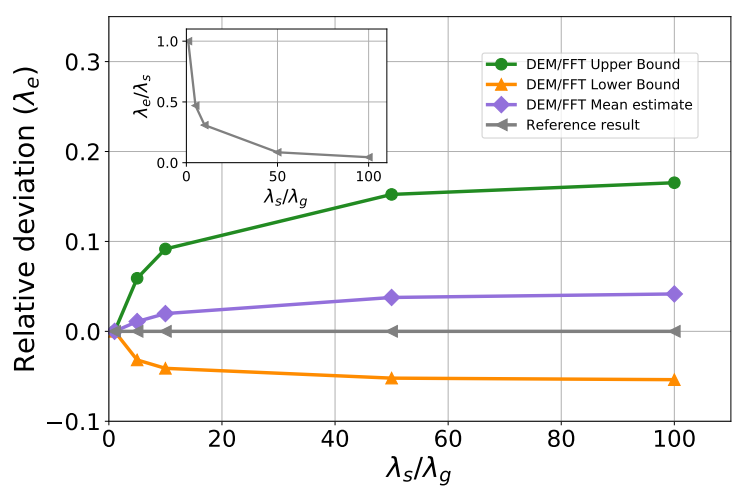

Figure 7: Effective conductivity of a solid made of identical spheres arranged on a periodic BCC array: deviation of the FFT computation $\left(r_{e q} / d l=30\right)$ compared to reference results (FFT, mean estimate, $\left.r_{e q} / d l=320\right)$.

\section{Results}

\subsection{DEM simulation of the packed beds}

Several packed beds are generated using the DEM. The gas volume fraction $(\approx 40.5 \%)$ as well as the size distribution of the polyhedra are based on the experimental data provided by Boegli [14] and described in details in section 2. Each generated granular medium contains 5000 particles with sizes ranging from 20 to $120 \mu \mathrm{m}$. Because the dispersion of the simulated ETC varies with the number of particles (or equivalently the size of the RVE) a specific sensitivity study to this parameter was conducted. This analysis revealed that this dispersion is stable when the number of particles exceeds 3000 and becomes very low $(\leq 3.5 \%)$ when this number reaches 5000 . The impact of the shape of particles was also analysed by increasing the number of faces from 4 to 20 of polyhedra. It was demonstrated that this parameter had no significant effect on the granular media ETC (less than $3 \%$ at $1000 \mathrm{~K}$ ). 
The RVE discretization parameter $d l$ (voxel size) considered is $2 \mu m$ (RVEs were constituted of nearly one billion voxels each). Simulated ETC bound may be more precise by decreasing the size of voxels to $1 \mu \mathrm{m}$ or less. However, in addition to increasing severely the intensity of FFT computations, considerations of the surface roughness of particles limit the physical validity of any further refinement of the discretization approaching the surface roughness. In [13], the surface roughness is estimated to be around $1 \mu \mathrm{m}$. Therefore, to remain conservative, no voxel size below $2 \mu \mathrm{m}$ is considered. In that sense, the provided FFT results allow to bound the uncertainty related to this physical feature of the considered bed. Considering a lower voxel size will require to represent more precisely these surface irregularities which is beyond the scope of this study.

The average size distribution of the particles for the different granular media generated with the DEM is reported on figure (8). The error bars represent the standard deviation between the five generated RVEs.

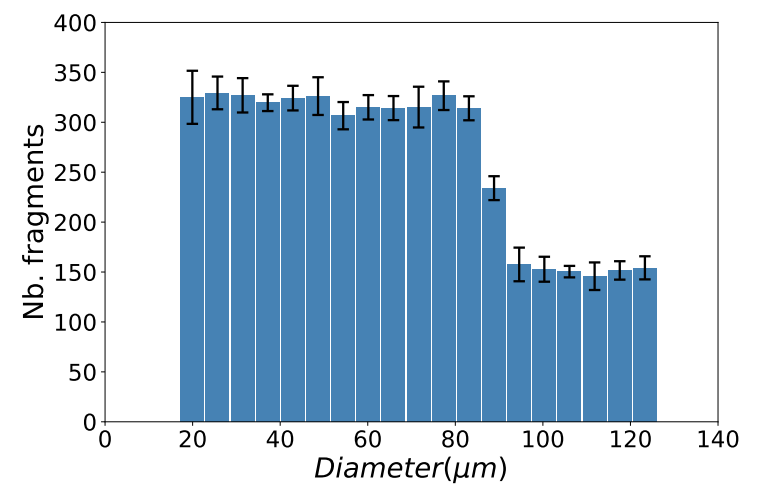

Figure 8: Size distribution of the polyhedra: the diameter of a polyhedron corresponds to the one of the sphere having the same volume. The distribution chosen is here representative of experimental packed beds of Boegli [14] with $60 \%$ of particles with diameters between 20 and $80 \mu \mathrm{m}$ and $40 \%$ between 80 and $120 \mu \mathrm{m}$. Without more detailed information, the polyhedra distributions in these ranges are arbitrarily chosen as equiprobable distributions in our simulations.

The distribution of particle size is known to affect the porosity of the generated media hence, in some cases, impacting greatly the ETC. The packed beds porosity can be partially controlled by modification of the Coulomb friction coefficient.

As the voxel size $d l$ equals $2 \mu m$, the discretization ratio $r_{e q} / d l$ varies from 10 (for the smallest particles) up to 60 (for the largest particles) which ensures that the relative volume error does not exceed $2 \%$ for any of the 5000 particles considered here.

The results of the DEM-FFT mean estimate and bounds of ETC as defined in Table 2, are reported in Fig. 9 as a function of the temperature and in Fig. 10 as a function of the solid to gas conductivity ratio. The mean-estimate no-contact appears in Fig. 9 and will be discussed in section 4.2. To lighten FFT computations results depicted on figures, only the averages of the computed ETC over the five RVEs are reported (the deviation between the ETC of the different RVEs and the mean value is less than $3.5 \%$ ). The lower bound is found to slightly underestimate the ETC of the packed bed for any conductivity ratio. The DEM-FFT upper bound possesses limited value at high conductivity contrasts where a large deviation between bounds is observed. This suggests the prominent role solid-gas and solidsolid interfaces on the ETC and the limits of the simple bounding method. As the discretization may not be refined further for physical reasons detailed above, the DEM-FFT bounds are not able to give adequate bounding of the ETC at high conductivity contrasts.

In addition, the mean estimate does not capture the expected increase of the ETC with temperature. To allow for consistent estimations of ETC, the mean estimation is improved in the next sections by having interfaces better modelled by considering the Knudsen effect as well as imperfections at solid-solid contacts notably in relation to the surface roughness of particles.

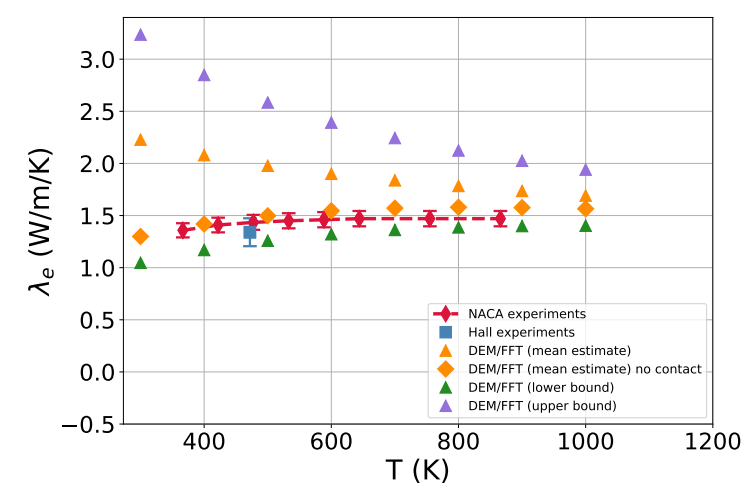

Figure 9: Evolution of the effective thermal conductivity as a function of temperature: simulation results compared to Hall experiments [13] and NACA experiments [14].

\subsection{Effect of the solid-solid interfaces on the ETC}

On Fig. 11 and Fig. 12, bimodal heat flux distributions for conductivity ratios $\lambda_{s} / \lambda_{g}=11$ and $\lambda_{s} / \lambda_{g}=65$ (lower bound) have been reported.

Even with the implementation of a contact resistance at solid-gas interfaces (lower bound: assignment of gas conductivity to interface voxels), heat fluxes in the 


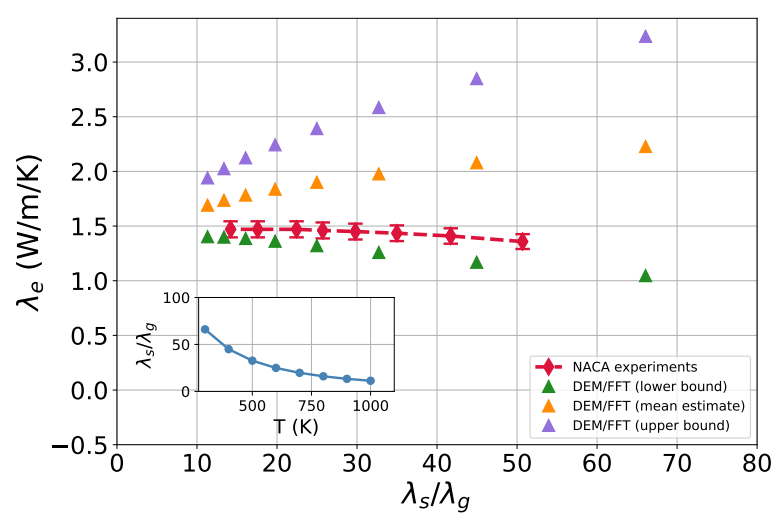

Figure 10: Evolution of the effective thermal conductivity as a function of the ratio $\lambda_{s} / \lambda_{g}(d l=2 \mu m)$ : simulation results compared to Hall experiments [13] and NACA experiments [14].

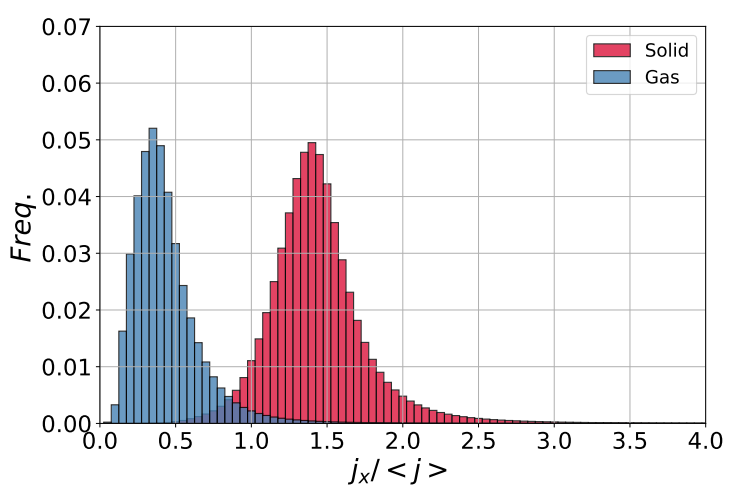

Figure 11: Heat fluxes (X-component) distribution. The heat fluxes are normalized by their volume average on the $\operatorname{RVE}\left(\lambda_{s} / \lambda_{g}=11\right.$, lower bound and $d l=2 \mu m$ ). gas phase remain significantly lower than ones in the solid phase especially at higher conductivity contrasts. The modelling of close contact areas between particles hence becomes critical in the simulation of the ETC of the RVE.

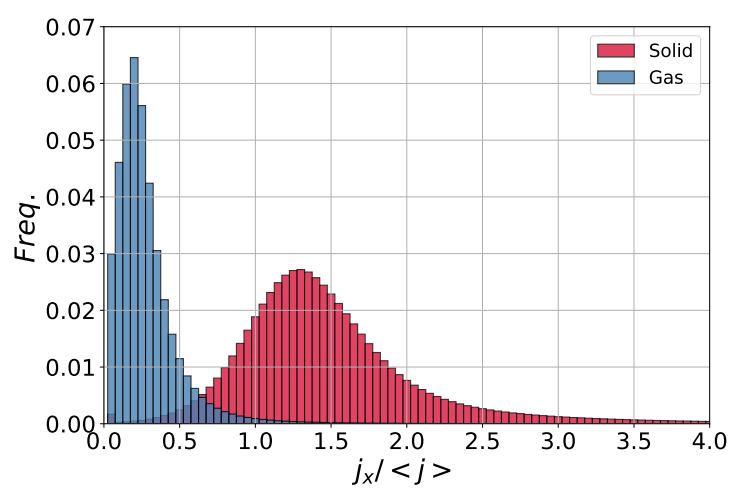

Figure 12: Heat fluxes (X-component) distribution. The heat fluxes are normalized by their volume average on the $\operatorname{RVE}\left(\lambda_{s} / \lambda_{g}=65\right.$, lower bound and $d l=2 \mu \mathrm{m}$ ).

When discretizing RVEs (especially during the discretization for the upper bound and to a lesser extent the mean estimate), some particles will be discretized so that they may be in direct contact with other particles in the discretized RVE. The presence of these perfect contacts, although limited in size and numbers, leads to overestimating the ETC especially at high conductivity contrasts. In real packed beds or even in the DEM generated packed-beds, the particles are almost always separated by a thin layer of gas and physical contacts are solely point contacts. These contacts points depends on roughness and are imperfect. They should be associated with a thermal contact resistance.

Therefore, solid-solid interfaces have been identified right after the discretization of our DEM generated medias. The value of the Coulomb friction coefficient of 0.2 between particles used in DEM computation, implies strong contact forces, in comparison to the packed bed global stress. This causes contacts to be mostly "vertex to face", "edge to edge" or "edge to face" contacts (point contact) and negligible "face to face" or "vertex to vertex" contacts between particles (see Fig. 13). Particles at contact areas have then been insulated from each other by a thinnest possible layer of gas so that heat fluxes may not pass through close contacts areas within going through at least one voxel of gas. The effect of solid-solid contacts is especially important at high conductivity contrasts. If two voxels from different solid particles found themselves adjacent, a non- 
realistic, solid thermal bridge is created during the discretization of the DEM generated packed bed. These thermal bridges, without being accounted for, consequently impact the simulated ETC, which is overestimated significantly at high phase conductivity contrasts, as shown in Fig. 9 and 10.

Considering the particle contacts insulation yields results depicted on Fig. 9. The Knudsen effect is not taken into account here but will be considered in the next section 4.3 .

The mean estimate with insulation of particles contacts is denoted "mean estimate no-contact" on Fig. 9. This estimate now follows the experimental trend curve as does the lower bound trend curve where the ETC is primarily driven by the fluid thermal conductivity. This trend is therefore similar to the evolution of Helium conductivity with temperature, depicted in Fig. 1. This result confirms the sensitivity of the ETC to the contribution of solid contacts between particles and provide a more accurate estimation in this case. However, this result should be considered with caution, as the solid/solid contact insulation is clearly an extremum of contact effective properties. The real contact conduction is certainly between gas and solid conductivity and depending on several parameters like contact force or roughness.

\subsection{Impact of the Knudsen effect on the ETC}

The significant deviation of bounds (Fig. 9 and 10) shows the importance of the solid-gas and solid-solid interfaces on the effective thermal conductivity notably at high conductivity contrasts. As depicted in figure 10 , the upper bound reaches three times the value of the lower bound. For the same contrast $\left(\lambda_{s} / \lambda_{g} \approx 65\right)$, the DEM-FFT bounds relative difference is significantly lower for the one computed in section 3.3 for the ideal BCC structure (22\% in Fig. 7). Although the deviation of bounds is lower, which may principally be explained by the difference in microstructure, the deviation still becomes consequent for the reference BCC structure.

As the proposed methodology models explicitly its micro-structure, the thermal fields through the granular media can also be studied. On Fig. 13 is reported a typical view of the heat flux field through the RVE. The temperature gradient is along $\mathrm{X}$ axis (horizontal on Fig. 13) while the contrast $\lambda_{s} / \lambda_{g}=8.2$. Here, the lower bound has been chosen for display. The particles can be easily identified on this figure as they correspond to high values of the heat flux while the gas corresponds to the lowest values. Consistently, the highest values of the heat flux are localized at close contact areas between particles. This last result illustrates the key role

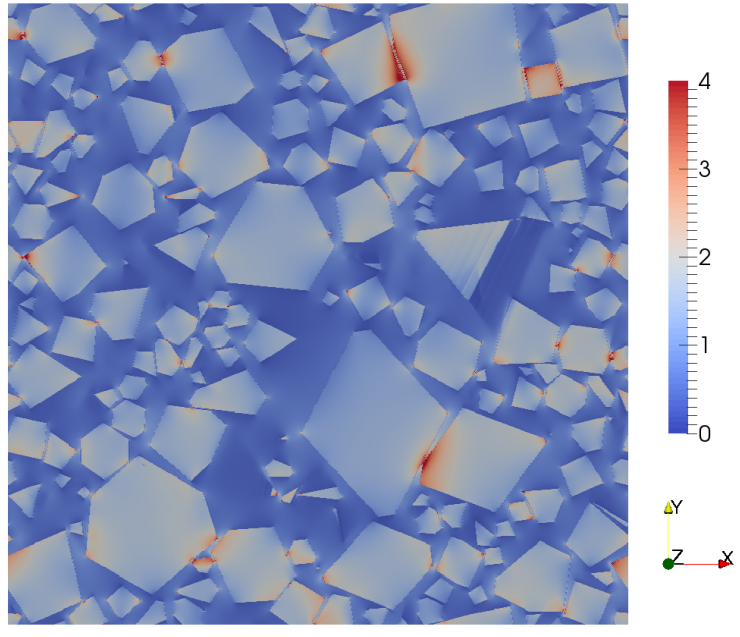

Figure 13: X-component of the simulated heat fluxes (lower bound) throughout the granular media (minimum and maximum values are blue and red, respectively). The heat fluxes are normalized by their volume average on the RVE. $\lambda_{s} / \lambda_{g}=8.2$ and $d l=2 \mu \mathrm{m}$.

of gas-solid interfaces on the ETC and therefore the necessity to consider the Knudsen effect in confined areas between particles.

If the thickness of the gas regions between particle is small (less than $10 \mu \mathrm{m}$ ), the Knudsen effect on the gas conductivity in these gas layers may not be negligible (see section 2). To study quantitatively this effect in a given voxelized microstructure, we chose to take it into account for all the gas voxels. To do so we have first to evaluate the distance between the solid voxels surrounding the gas voxels considered. This computation is equivalent to evaluate, for each gas voxel, the size of the cavities in the packed bed: the pore size. The local pore size attached to each voxel is computed thanks to the so-called "Maximum inscribed ball" (see [25]) technique which consists in finding the largest inscribed spheres centered on each voxel of the gas space that just touches the solid surface. Those that are fully overlapped by larger spheres are removed. The remaining spheres are called maximal balls which covers fully the gas space. For the packed beds considered hereafter, this pore size distribution will be computed thanks to this maximum inscribed balls technique (see Fig. 14).

Then, for each gas voxel, the thickness of the gas layer is defined hereafter as the diameter of the maximum inscribed ball to which the voxel belongs. Thus, as the size of the confined space $L$ (the thickness of the gas layer) is defined for each voxel, the associated Knudsen number $K_{n}$ can be computed from relation (4). Here, 


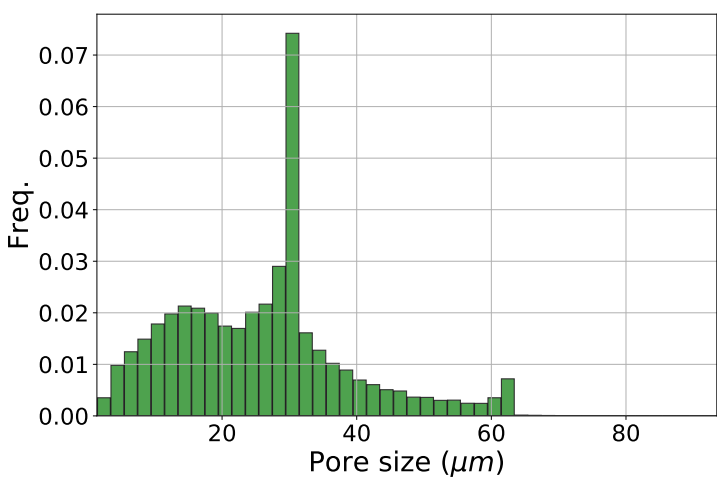

Figure 14: Size distribution of the diameter of the maximum inscribed balls (gas voxels) in the considered packed bed $(d l=2 \mu \mathrm{m})$.

following [11], the corrected effective thermal conductivity of the considered voxel gas is finally estimated by :

$$
\lambda_{g}^{c}=\frac{\lambda_{g}}{1+2 \beta K_{n}}
$$

where $\beta$ is given by:

$$
\beta=\frac{2-\alpha_{c}}{\alpha_{c}} \quad \text { and } \quad \alpha_{c}=\frac{C m_{r}}{\left(1+m_{r}\right)^{2}}
$$

with $C=2.4$, an empirical constant, $m_{r}=m_{g} / m_{s}$ the ratio of the gas $\left(m_{g}\right)$ to the solid $\left(m_{s}\right)$ atomic masses. Even under the high pressure adopted by [14] (1.7 MPa) for their reported experimental data and for a confinement distance equal to $10 \mu \mathrm{m}$, this correction leads to a decrease of $12 \%(300 \mathrm{~K})$ to $31 \%(1000 \mathrm{~K})$ of the conductivity of the gas. This correction will be systematically applied to each gas voxel to take into account the Knudsen effect.

\section{Discussion}

The heat-flux maps reported on the previous Fig. 13 have shown the key-role of the gas regions localized between particles on the heat propagation through the packed bed. Hence, on Fig. 15, we have reported the lower FFT bound and mean estimates of the ETC taking into account the Knudsen correction.

As expected, the effect of the Knudsen correction on the effective conductivity increases with temperature. In addition and even if the volume fraction of gas voxels concerned by this correction is relatively low (about $10 \%$ ), we can observe on this figure that the correction can no longer be ignored (about $20 \%$ at $1000 \mathrm{~K}$ ). As expected, experimental data are located between the

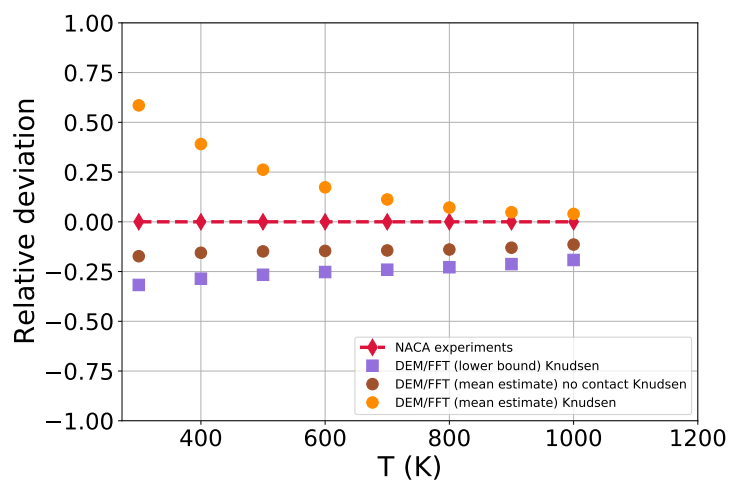

Figure 15: Relative deviation of the simulated ETC as a function of the temperature: simulated results $(d l=2 \mu m)$ with Knudsen.

bounds. In addition, and thanks to this Knudsen correction, experimental data are now better surrounded by the upper and lower estimates (denoted by "mean estimate Knudsen" and "mean estimate no-contact Knudsen", respectively).

Solid to solid thermal resistances at contact points between particles are also known to decrease the ETC of packed beds alongside the Knudsen effect. However, with the discretization parameter ('dl') selected in this study, and given the phases conductivity ratio, the contact thermal resistance should be lesser than the separation of particles with gas voxels accompanied with the Knudsen effect, hence allowing to still bound the experimental ETC. A more accurate modelling of this thermal contact resistance may allow to further narrow the difference obtained between experimental and simulated results. Improved modelling of the Knudsen effect and contacts between particles combined with potential effects of particle roughness and fluid pressure should be further studied to allow for better estimates of the ETC, especially for more complex packed beds, for instance possessing higher phase conductivity contrasts.

Even though the mean estimate no-contact yields the most accurate results, it should be considered as the reference DEM-FFT as the trend of this estimation (cf. Fig. 15) adequately follows the experimental trend with temperature. It is important to note that this DEM-FFT mean estimate no-contact has the most constant deviation among all models. The predicted results are however below the experimental data point (relative difference of about $15 \%$ ). This may mainly be explained by the contact correction which may be, in some high heat flux contact areas, too significant. Particles are insulated from each other by at least $2 \mu \mathrm{m}$ which is usually, in critical areas for heat transfers, larger than the true dis- 
tance separating particles. This may be improved by assigning a corrected thermal conductivity to contact area voxels based on the DEM generated media distances between particles, ideally also dependent on the direction of the heat flux.

Additionally, the effect of the chosen correction (the choice of the empirical constant $C$ in equation (11) for instance) on this trend may have impact on the final result, and allow for more accurate framing of ETC results but this sensitivity analysis is left for future works.

\section{Conclusions}

In this work, a methodology based on the DEM (generation of RVEs) and FFT (calculation of effective properties) to simulate through bounds and estimations the ETC of granular media made of solid particles embedded in a stagnant fluid was proposed.

- The DEM-FFT method is an efficient method for computing the ETC of granular media when no or negligible radiation is involved. Identification of key voxels for the media ETC may be detected rapidly and their respective thermal conductivity may be corrected. This method although used here for a simple dual phase media may be used for more complex media involving multiple anisotropic phases or other complex composites.

- Sensitivity analyses using the DEM-FFT method allow to determine the optimal RVE size, number of considered particles and discretization size (ie. 5000 particles and nearly 1 billion voxels size RVEs). The maximal discretization was herein determined by the particle surface roughness determined in reference experimental work.

- DEM-FFT lower and upper bounds can be constructed by identifying voxels of interfaces and the assignment of extreme properties. These bounds may effectively surround experimental data. However, at high conductivity contrasts, significant deviation of the bounds is seen. This deviation demonstrates the prominent role of the solid-gas and solid-solid interfaces on the ETC of the granular bed.

- The DEM-FFT usual estimate called "mean estimate" in this paper is not able to capture the effect of the temperature on the evolution of the ETC of the granular bed under consideration. Indeed, special care and correction to the thermal conductivity solid-solid interfaces are necessary to effectively predict the ETC of granular media. Considering the inclusion of a thin gas insulation layer in contact areas, allows for the proposal of a new lower estimate (called "mean estimate no-contact") following the experimental data trend.

- Our "mean estimate no-contact" complemented with a Knudsen correction is the proposed reference model to estimate the ETC of granular media. Although, slightly underestimating the experimental measurement, it provides a consistent estimation of ETC for all conductivity contrasts. Among all models, it has the most constant difference with reference ETC measurements.

- In future works, a better DEM/FFT modelling of particle surface roughness as well as an improved modelling of contact interfaces will allow for a closer framing of experimental data. Granular media with larger particle ratio and contrast between solid and gas conductivity may be studied to prove the robustness of the method and considerations of the contribution of radiation using the already implemented inscribed ball technique may allow to predict the conductivity where all mechanisms of heat transfer are present.

\section{Acknowledgements}

The authors acknowledge Dr. E. Castelier for his support in working with TMFFT software (version 1.8). The authors also acknowledge the financial support of the French Alternative Energies and Atomic Energy Commission (CEA), EDF and FRAMATOME.

\section{References}

[1] O. Wiener. Die theorie des mischk orpers fur das feld der station aren stromung. Abhandlungen der Sachsischen Gesellschaft der Akademischen Wissenschaften in Mathematik und Physik, 32:507-604, 1912

[2] J. C. Maxwell. A treatise on electricity and magnetism, volume 1. Clarendon press, 1873.

[3] George Keith Batchelor and R. W. O'Brien. Thermal or electrical conduction through a granular material. Proceedings of the Royal Society of London. A. Mathematical and Physical Sciences, 355(1682):313-333, 1977.

[4] S. Imura and E. Takegoshi. Effect of gas pressure on effective thermal conductivity of packed beds. Transactions of the Japan Society of Mechanical Engineers, 40:489-497, 101974.

[5] M. Okazaki, M. Yamasaki, T. Gotoh, and S. Toei. Effective thermal conductivities of wet granular materials. In AIChE Symposium Series, volume 163, pages 164-176, 1977.

[6] L.O. Jernkvist and A.R. Massih. Models for axial relocation of fragmented and pulverized fuel pellets in distending fuel rods and its effects on fuel rod heat load. Swedish Radiation Safety Authority, 092015. 
[7] H.L. Xing H.W. Zhang, Q. Zhou and H. Muhlhaus. A dem study on the effective thermal conductivity of granular assemblies. Powder Technology, 205:172-183, 2011.

[8] L. Chen, C. Wang, M. Moscardini, M. Kamlah, and S. Liu. A dem-based heat transfer model for the evaluation of effective thermal conductivity of packed beds filled with stagnant fluid: Thermal contact theory and numerical simulation. International Journal of Heat and Mass Transfer, 132:331 - 346, 2019.

[9] T. S. Yun and T. M. Evans. Three-dimensional random network model for thermal conductivity in particulate materials. Computers and Geotechnics, 37(7):991 - 998, 2010.

[10] M. Guessasma H. Haddad and J. Fortin. Heat transfer by conduction using dem-fem coupling method. Computational Materials Science, 81:339-347, 2014.

[11] M. Moscardini, Y. Gan, S. Pupeschi, and M. Kamlah. Discrete element method for effective thermal conductivity of packed pebbles accounting for the smoluchowski effect. Fusion Engineering and Design, 127:192-201, 2018.

[12] L. Gillibert F. Willot and D. Jeulin. Microstructure-induced hotspots in the thermal and elastic responses of granular media. International Journal of Solids and Structures, 50(10):16991709,2013

[13] R.O.A. Hall, D.G. Martin, and M.J. Mortimer. The thermal conductivity of uo2 sphere-pac beds. Journal of Nuclear Materials, 173(2):130-141, 1990.

[14] J.S. Boegli and R.G. Deissler. Measured effective thermal conductivity of uranium oxide powder in various gases and gas mixtures. National Advisory Committee for Aeronautics Research Memorandum, 1955.

[15] P.G. Lucuta, H.J. Matzke, and I.J. Hastings. A pragmatic approach to modelling thermal conductivity of irradiated $u_{2}$ fuel: review and recommendations. Journal of nuclear materials, 232(2-3):166-180, 1996.

[16] R. Drut. Caractéristiques dynamiques de l'hélium: Formules et tables. Service central de documentation du CEA, CEA-R3791:1-35, 1968.

[17] T.T. Claudson. Emissivity data for uranium dioxide. Oregon State College, 1958

[18] M. Smoluchowski. On conduction of heat by rarefied gases. Pisma Mariana Smoluchowskiego, 1(1):139-155, 1924.

[19] F. Radjai and F. Dubois. Modélisation numérique discrète des matériaux granulaires, 2010.

[20] F. Dubois, M. Jean, M. Renouf, R. Mozul, A. Martin, and M. Bagneris. Lmgc90. 10e colloque national en calcul des structures. Giens, France, 2011.

[21] R. Hill. Elastic properties of reinforced solids: some theoretical principles. Journal of the Mechanics and Physics of Solids, 11(5):357-372, 1963.

[22] H. Moulinec and P. Suquet. A numerical method for computing the overall response of nonlinear composites with complex microstructure. Computer methods in applied mechanics and engineering, 157(1-2):69-94, 1998.

[23] H. Moulinec. A fast numerical method for computing the linear and nonlinear mechanical properties of composites. C. R Acad. Sci. Paris, 318:1417-1423, 1994.

[24] D.R. McKenzie, R.C. McPhedran, and G.H. Derrick. The conductivity of lattices of spheres-ii. the body centred and face centred cubic lattices. Proceedings of the Royal Society of London. A. Mathematical and Physical Sciences, 362(1709):211-232, 1978.

[25] A.N. Houston, W. Otten, R. Falconer, O. Monga, P.C. Baveye, and S.M. Hapca. Quantification of the pore size distribution of soils: assessment of existing software using tomographic and synthetic 3d images. Geoderma, 299:73-82, 2017. 